\title{
Diferencias biomecánicas del tiro libre en el fútbol entre jugadores del club Jit y Atahualpa
}

\author{
Oscar David Quilachamin Espinoza \\ odquilachamin@espe.edu.ec
}

Miguel Angel Torres Morillo matorres11@espe.edu.ec

Excehomo Gabriel Coral Apolo gecoral@espe.edu.ec

Afiliación institucional del equipo de investigación: Universidad de las Fuerzas Armadas - ESPE

\section{RESUMEN}

Quito - Ecuador

Introducción: La investigación se basa en estudiar las diferencias biomecánicas existentes entre jugadores de distinto nivel, como es el club JIT (Juventud Independiente de Tabacundo) perteneciente al fútbol de segunda categoría profesional de Pichincha y el club Sociedad Deportiva Atahualpa, que se encuentra en categoría Amateur del fútbol de Pichincha. Objetivo: Comparar las diferencias biomecánicas entre jugadores profesionales y amateurs en el tiro libre para determinar los movimientos que permiten tener mayor efectividad en el elemento técnico por medio del software Kinovea. Métodos: Para determinar los movimientos que influyen en la correcta ejecución del tiro libre se empleó el software Kinovea, analizando así cada gesto detalladamente, el uso de una cámara y un ordenador de calidad son necesarios para el estudio por ello se trabajó con equipos de estándares altos. Se analizaron dos muestras independientes con el software estadístico SPSS, empleando la prueba U de Mann-Whitney. Resultados: Se determina que, existe diferencias significativas entre las variables estudiadas, para obtener la mayor eficiencia del gesto técnico se debe formar un ángulo de apertura de piernas entre 75,66 ${ }^{\circ}$. También se debería tener una velocidad de $8,96 \mathrm{~m} / \mathrm{s}$ y un ángulo de la articulación de la rodilla de la pierna base aproximado en un $106,81^{\circ}$. Conclusiones: Las condiciones en las que se realizaron el estudio del tiro libre en el fútbol son iguales, lo que permite obtener datos verídicos. La mayor influencia se encuentra en el nivel técnico que tiene el jugador profesional en relación con el jugador amateur, con algunas excepciones.

Palabras clave: Biomecánica, tiro libre, fútbol. 


\title{
Biomechanical differences of free kick in soccer between players of Jit and Atahualpa clubs
}

\begin{abstract}
Introduction: The research is based on studying the existing biomechanical differences between players of different levels, as is JIT club (Juventud Independiente de Tabacundo) pertaining to the second professional category soccer of Pichincha and Sociedad Deportiva Atahualpa club, which is in Amateur category of Pichincha soccer. Objective: Compare the biomechanical differences between professional and amateur players in the free kick to determine the movements that permit greater effectiveness in the technical element using the Kinovea software. Methods: To determine the movements that influence the correct execution of the free kick, the Kinovea software was used, analyzing each gesture in detail, the use of a camera and a quality computer are necessary for the study, for this reason we worked with high standard equipment. Two independent samples were analyzed with SPSS statistical software, using the Mann-Whitney U test. Results: It is determined that there are significant differences between the variables studied, in order to achieve the greatest efficiency of the technical gesture, a leg opening angle of $75.66^{\circ}$ should be formed, as well as a speed of $8.96 \mathrm{~m} / \mathrm{s}$ and an angle of the knee articulation of the base leg of approximately $106.81^{\circ}$. Conclusions: The conditions in which the study of the free kick in soccer were performed are identical, which allows us to obtain truthful data. The greatest influence is found in the technical level that the professional player has in relation to the amateur player, with some exceptions.
\end{abstract}

Keywords: Biomechanics, free kick, soccer.

Artículo recibido: 10. Junio. 2021 Aceptado para publicación: 16. Julio. 2021 Correspondencia: odquilachamin@espe.edu.ec Conflictos de Interés: Ninguna que declarar 


\section{INTRODUCCIÓN}

Partimos con una definición de lo que engloba la biomecánica, muchos autores la definen como la ciencia que estudia los movimientos corporales, pero en relación con la parte deportiva se menciona que esta materia influye notablemente en el mejoramiento de la técnica deportiva debido a que ayuda comprender todo lo que conlleva la práctica deportiva y también al perfeccionamiento del entrenamiento. Ferro y Floría (2007).

Al encontrar una estrecha relación con el deporte varios autores han empleado el uso de esta ciencia y más en el análisis para deportes de alto rendimiento dado que además de entender todos los movimientos y variantes biomecánicas, favorece a desarrollar aplicaciones que potencialicen el gesto técnico y a su vez sirvan como medida preventiva para lesiones a partir del análisis. Mantilla (2019).

La biomecánica deportiva está enfocada en el estudio de la mecánica del sistema locomotor de los deportistas, entre los análisis de la biomecánica esta la estructura corporal de la persona, la ejecución correcta del gesto técnico, etc. Páez (2010).

De igual manera la aplicación de la biomecánica como parte de la actividad física y el deporte enfocada a la investigación de los movimientos de los atletas tiene como objetivo la evaluación de los distintos gestos deportivos, analizando la práctica deportiva para mejorar su rendimiento, desarrollar técnicas de entrenamiento y diseñar complementos, materiales y equipamiento de altas prestaciones. Perdomo, Pegudo y Capote (2018).

El estudio del movimiento permite a los investigadores encontrar detalles influyentes para una correcta ejecución del gesto técnico, en la presente investigación se analizó el tiro libre en el fútbol, es importante analizar las diferencias en dos distintos grupos, con un nivel técnico diferente para así obtener resultados verídicos y que contribuyan al deporte.

Aplicar la biomecánica específicamente en el fútbol, permite localizar distintos indicadores los cuales influirán en la buena ejecución de los elementos técnicos a estudiar, entre ellos se encuentran: ángulo de la articulación femorotibial antes, durante y después de tener contacto con el balón, ángulos de aperturas de piernas, velocidad de la pierna ejecutante del tiro libre en el fútbol. Jordán, Espinoza, Aguilar, Hidalgo y Gutiérrez (2018).

Para la investigación se emplea el software Kinovea que es un programa de video análisis para diferentes deportes y el cuál tiene diversas herramientas para el estudio de elementos técnicos que son parte de cada disciplina deportiva. (Charmant, 2004). 
Las herramientas del Software Kinovea que fueron empleadas son: Análisis de velocidad, ángulos para determinar la apertura de las piernas y el goniómetro el cual es un instrumento que permite realizar una medición del arco de movimiento de articulaciones del cuerpo, Angos (2015).

También se utiliza el software estadístico SPSS el cual es reconocido a nivel mundial por su gran utilidad de trabajo con grandes bases de datos, el software estadístico es sumamente importante en el trabajo ya que permite trabajar con el área de Ciencias Humanas y Sociales. Fernández (2017).

La investigación fue realizada con un club profesional, definido por Rodríguez (2011) como: "Entidad dedicada a un uso deportivo, situación por la cual ostenta equipos e instalaciones que están destinados a la práctica de uno o más deportes." Como se menciona el club profesional tiene la facilidad de entrenar a diario, tienen los recursos necesarios para mejorar el aspecto físico y técnico de sus jugadores, además cada jugador recibe un sueldo por lo cual se dedican de lleno al fútbol.

Por otro lado, tenemos al club del fútbol amateur, Núñez y Briones (2015) menciona que: "el fútbol amateur es practicado principalmente los fines de semana, sin mayor exigencia deportiva." Estos tipos de clubes tienen más complicaciones, los jugadores en esta categoría lo ven como una forma de distracción y amor a la práctica deportiva, ya que no reciben remuneración alguna, entrenan máximo tres días por semana lo que impide el crecimiento futbolístico. Muchos de estos jugadores tienen un trabajo aparte y estable lo que hace que no dependan del entrenamiento en el deporte. Aquí la importancia decae sobre la confianza que le inyecte el entrenador al jugador para que continúe con la práctica del fútbol, es importante la parte psicológica para no dejar a un lado el entrenamiento, así mismo como la motivación y el trabajo en equipo mejorando la relación del cuerpo técnico. Roffé (2015).

El objetivo primordial de la investigación es comparar las diferencias biomecánicas entre jugadores profesionales y amateurs en el tiro libre para determinar los movimientos que permiten tener mayor efectividad en el elemento técnico por medio del software Kinovea. MÉTODOS

La presente investigación es de tipo correlacional ya que como menciona Mejía (2019): "Es un tipo de investigación no experimental en la que los investigadores miden dos variables y establecen una relación estadística entre las mismas (correlación), sin necesidad 
de incluir variables externas para llegar a conclusiones relevantes." El análisis biomecánico fue realizado con el programa Kinovea, el cual es un software de libre acceso que permite el visionado de imágenes y video, compatible con el sistema operativo Windows y disponible en cerca de 15 idiomas. Sánchez (2018). Por tal motivo se estudiaron dos grupos distintos. El primer grupo analizado fue el club deportivo JIT (Juventud Independiente de Tabacundo), en el cual se analizaron 15 jugadores entre edades de 19 y 28 años de distintas posiciones de juego en el campo de fútbol. EL otro grupo estudiado, fue el club Sociedad Deportiva Atahualpa, igualmente fueron 15 jugadores entre edades de 18 y 26 años de diferentes posiciones.

Se realizó la grabación de cada tiro libre de todos los jugadores con el celular Samsung Edge 7 que tiene una cámara de Dual Pixel 12.0 MP y una resolución de grabación de vídeo UHD 4K (3840 x 2160) @30fps lo cual permite tener buenas tomas del elemento técnico. (Samsung, 2016)

Según Vásquez y Montesinos (2016) menciona que: "En fútbol, un tiro libre es una forma de reanudar el juego tras una falta. El tiro libre directo es un cobro directo a la portería del equipo contrario.” En este caso se analizó un tiro libre directo, ejecutado en cancha de césped, y en arcos de fútbol profesionales, con una distancia aproximada de $25 \mathrm{~m}$, la posición del balón varío en dependencia de la pierna hábil de cada jugador o de la preferencia de este.

Posterior a la grabación, se guardaron los videos en un ordenador DELL, en donde se estudiaron los videos y el cual tiene características que permiten un buen análisis como pantalla full HD de 15.6 pulg, Radeon Graphics e Intel Core i7 lo que permite que se reproduzcan videos fácilmente. (Dell, s.f.)

\section{Las variables estudiadas fueron:}

1) Velocidad máxima del deportista al realizar el tiro libre: En esta variable se estudia la velocidad del pie de ejecución al impactar con el balón en el tiro libre, para esto se trazó la trayectoria desde la fase inicial del pateador hasta la fase final, observando detalladamente la velocidad en la fase de golpeo. Los resultados son expresados en m/s.

2) Ángulos de apertura de las piernas al momento de la ejecución del tiro libre: Para esta variable se toman como referencia tres puntos: el pie de apoyo, el pie que impacta con el balón y la cadera del jugador. Ubicados estos puntos se puede observar la apertura que tiene un pie del otro, sus resultados están expresados en ángulos. 
3) Ángulo de la articulación de la rodilla de la pierna base: En esta variable se estudia el ángulo que adopta la rodilla al momento en el que el deportista ejecuta el tiro libre. Se toma como referencia los siguientes tres puntos: Pie base del jugador, rodilla de la pierna base y articulación de la cadera.

Con estas tres variables obtenidas se procede a transcribir los datos en el software estadístico SPSS en el cual se utilizó el análisis de datos para dos muestras independientes empleando la prueba $U$ de Mann-Whitney $(\mathrm{p} \leq 0.05)$, para comprobar si existe diferencia significativa.

\section{RESULTADOS Y DISCUSIÓN}

Tabla 1: Datos de velocidad obtenidos al ejecutar el tiro libre del club JIT (Grupo 1)

\begin{tabular}{|c|c|c|}
\hline$\#$ & Datos & Grupos \\
\hline Deportista 1 & 6,52 & 1 \\
\hline Deportista 2 & 5,59 & 1 \\
\hline Deportista 3 & 9,24 & 1 \\
\hline Deportista 4 & 6,58 & 1 \\
\hline Deportista 5 & 9,54 & 1 \\
\hline Deportista 6 & 13,71 & 1 \\
\hline Deportista 7 & 8,83 & 1 \\
\hline Deportista 8 & 12,21 & 1 \\
\hline Deportista 9 & 12,63 & 1 \\
\hline Deportista 10 & 8,56 & 1 \\
\hline Deportista 11 & 17,47 & 1 \\
\hline Deportista 12 & 7,44 & 1 \\
\hline Deportista 13 & 5,30 & 1 \\
\hline Deportista 14 & 5,32 & \\
\hline Deportista 15 & 5,43 & 134,7 \\
\hline Suma total: & \multicolumn{2}{|c|}{} \\
\hline Valor máximo: & \multicolumn{2}{|c|}{8,47} \\
\hline Valor mínimo: & \multicolumn{2}{|c|}{} \\
\hline Promedio:
\end{tabular}

Como se puede observar el promedio de velocidad del pie al impactar con el balón es de $8,96 \mathrm{~m} / \mathrm{s}$, cabe resaltar que el jugador número 11 de la tabla 1 tiene la mayor velocidad de ejecución esto ya que su tiro es potente y busca las esquinas del arco, por otro lado, el jugador número 13 de la tabla 1 tiene una velocidad de 5,3 el cual es el valor mínimo de toda la muestra tomada, esto se puede dar por que su tiro no llevaba potencia sino más bien estaba enfocado a un tiro libre de colocación. 
Tabla 2: Datos de velocidad obtenidos al ejecutar el tiro libre del club Atahualpa (Grupo 2)

\begin{tabular}{|c|c|c|}
\hline \# & Datos & Grupos \\
\hline Deportista 1 & 5,29 & 2 \\
\hline Deportista 2 & 4,50 & 2 \\
\hline Deportista 3 & 5,18 & 2 \\
\hline Deportista 4 & 6,70 & 2 \\
\hline Deportista 5 & 3,31 & 2 \\
\hline Deportista 6 & 6,13 & 2 \\
\hline Deportista 7 & 2,37 & 2 \\
\hline Deportista 8 & 6,78 & 2 \\
\hline Deportista 9 & 5,08 & 2 \\
\hline Deportista 10 & 7,34 & 2 \\
\hline Deportista 11 & 6,15 & 2 \\
\hline Deportista 12 & 4,93 & 2 \\
\hline Deportista 13 & 5,47 & 2 \\
\hline Deportista 14 & 7,94 & 2 \\
\hline Deportista 15 & 6,89 & 2 \\
\hline Suma total: & \multicolumn{2}{|c|}{84,06} \\
\hline Valor máximo: & \multicolumn{2}{|c|}{7,94} \\
\hline Valor mínimo: & \multicolumn{2}{|c|}{2,37} \\
\hline Promedio: & \multicolumn{2}{|c|}{5,60} \\
\hline
\end{tabular}

En el segundo grupo se puede evidenciar que el promedio de velocidad del equipo es de $5,60 \mathrm{~m} / \mathrm{s}$. En el equipo Atahualpa el jugador número 14 de la tabla 2 obtuvo una velocidad alta la cual es 7,94. Por otro lado, la velocidad mínima fue de 2,37 obtenida por el jugador número 7 de la tabla 2.

Tabla 3: Datos de angulos de apertura recogidos al ejecutar el tiro libre del club JIT (Grupo 1)

\begin{tabular}{|c|c|c|}
\hline$\#$ & Datos & Grupos \\
\hline Deportista 1 & 62,00 & 1 \\
\hline Deportista 2 & 74,00 & 1 \\
\hline Deportista 3 & 72,00 & 1 \\
\hline Deportista 4 & 72,00 & 1 \\
\hline Deportista 5 & 69,00 & 1 \\
\hline Deportista 6 & 75,00 & 1 \\
\hline Deportista 7 & 72,00 & 1 \\
\hline Deportista 8 & 78,00 & \\
\hline
\end{tabular}




\begin{tabular}{|l|c|c|}
\hline Deportista 9 & 92,00 & 1 \\
\hline Deportista 10 & 72,00 & 1 \\
\hline Deportista 11 & 95,00 & 1 \\
\hline Deportista 12 & 72,00 & 1 \\
\hline Deportista 13 & 75,00 & 1 \\
\hline Deportista 14 & 77,00 & 1 \\
\hline Deportista 15 & 78,00 & 1 \\
\hline Suma total: & \multicolumn{2}{|c|}{1135} \\
\hline Valor máximo: & \multicolumn{3}{|c|}{62} \\
\hline Valor mínimo: & \multicolumn{3}{|c|}{75,66} \\
\hline Promedio:
\end{tabular}

En esta variable de ángulos de apertura se puede observar que un promedio del equipo está en los $75,66^{\circ}$, podemos evidenciar que el valor máximo es de $95^{\circ}$ del jugador número 11 de la tabla 3 quien obtuvo una velocidad alta en los resultados de la tabla número 1, por otro lado, el menor ángulo obtenido es $62^{\circ} \mathrm{el}$ cual fue el resultante del jugador número 1.

Tabla 4: Datos de angulos de apertura recogidos al ejecutar el tiro libre del club Atahualpa (Grupo 2)

\begin{tabular}{|c|c|c|}
\hline \# & Datos & Grupos \\
\hline Deportista 1 & 65,00 & 2 \\
\hline Deportista 2 & 76,00 & 2 \\
\hline Deportista 3 & 67,00 & 2 \\
\hline Deportista 4 & 45,00 & 2 \\
\hline Deportista 5 & 77,00 & 2 \\
\hline Deportista 6 & 79,00 & 2 \\
\hline Deportista 7 & 73,00 & 2 \\
\hline Deportista 8 & 49,00 & 2 \\
\hline Deportista 9 & 45,00 & 2 \\
\hline Deportista 10 & 53,00 & 2 \\
\hline Deportista 11 & 50,00 & 2 \\
\hline Deportista 12 & 47,00 & 2 \\
\hline Deportista 13 & 45,00 & 2 \\
\hline Deportista 14 & 67,00 & 2 \\
\hline Deportista 15 & 65,00 & 2 \\
\hline Suma total: & \multicolumn{2}{|c|}{903} \\
\hline Valor máximo: & \multicolumn{2}{|c|}{79} \\
\hline Valor mínimo: & \multicolumn{2}{|c|}{45} \\
\hline Promedio: & \multicolumn{2}{|c|}{60,02} \\
\hline
\end{tabular}


En esta variable estudiada se observa que el promedio de ángulo del club Atahualpa es de $60,02^{\circ}$, su valor máximo fue $79^{\circ}$ obtenido por el jugador número 6 de la tabla 4 el cual en su resultado de velocidad también obtuvo un buen puntaje dejándonos claro que, haciendo un promedio entre los dos grupos, un ángulo estándar para la ejecución del tiro libre es $67,84^{\circ}$, por otro lado, el menor ángulo fue obtenido por el jugador número 9 de la tabla 4 con un valor de $45^{\circ}$.

Tabla 5: Datos de angulos de la rodilla de la pierna base al ejecutar el tiro libre del club JIT (Grupo 1)

\begin{tabular}{|c|c|c|}
\hline$\#$ & Datos & Grupos \\
\hline Deportista 1 & 154,30 & 1 \\
\hline Deportista 2 & 40,50 & 1 \\
\hline Deportista 3 & 59,50 & 1 \\
\hline Deportista 4 & 114,20 & 1 \\
\hline Deportista 5 & 120,80 & 1 \\
\hline Deportista 6 & 126,40 & 1 \\
\hline Deportista 7 & 131,50 & 1 \\
\hline Deportista 8 & 107,30 & 1 \\
\hline Deportista 9 & 98,20 & 1 \\
\hline Deportista 10 & 100,30 & 1 \\
\hline Deportista 11 & 102,90 & 1 \\
\hline Deportista 12 & 106,00 & 1 \\
\hline Deportista 13 & 110,00 & 1 \\
\hline Deportista 14 & 128,00 & 1 \\
\hline Deportista 15 & 102,30 & 1 \\
\hline Suma total: & \multicolumn{2}{|c|}{1602,2} \\
\hline Valor máximo: & \multicolumn{2}{|c|}{154,3} \\
\hline Valor mínimo: & \multicolumn{2}{|c|}{40,5} \\
\hline Promedio: & \multicolumn{2}{|c|}{106,81} \\
\hline
\end{tabular}

En esta variable estudiada se observa que el promedio de ángulo de rodilla de la pierna base del grupo 1 es de $106,81^{\circ}$, su valor máximo fue $154,3^{\circ}$ obtenido por el jugador número 1 de la tabla 5, el valor mínimo lo obtiene el jugador número 2 de la tabla 5 con un ángulo de $40,5^{\circ}$, que se traduce también a una menor velocidad al momento de ejecutar el tiro libre.

Tabla 6: Datos de angulos de la rodilla de la pierna base al ejecutar el tiro libre del club Atahualpa (Grupo 2) 


\begin{tabular}{|c|c|c|}
\hline$\#$ & Datos & Grupos \\
\hline Deportista 1 & 143,30 & 2 \\
\hline Deportista 2 & 119,40 & 2 \\
\hline Deportista 3 & 116,00 & 2 \\
\hline Deportista 4 & 124,40 & 2 \\
\hline Deportista 5 & 112,00 & 2 \\
\hline Deportista 6 & 127,90 & 2 \\
\hline Deportista 7 & 114,70 & 2 \\
\hline Deportista 8 & 129,70 & 2 \\
\hline Deportista 9 & 141,00 & 2 \\
\hline Deportista 10 & 142,90 & 2 \\
\hline Deportista 11 & 140,50 & 2 \\
\hline Deportista 12 & 128,00 & 2 \\
\hline Deportista 13 & 132,70 & 2 \\
\hline Deportista 14 & 124,00 & 2 \\
\hline Deportista 15 & 139,80 & 2 \\
\hline Suma total: & \multicolumn{2}{|c|}{1936,3} \\
\hline Valor máximo: & \multicolumn{2}{|c|}{143,3} \\
\hline Valor mínimo: & \multicolumn{2}{|c|}{112} \\
\hline Promedio: & \multicolumn{2}{|c|}{129,09} \\
\hline
\end{tabular}

En esta variable analizada se observa que en el grupo 2 existe un promedio de 129,09 mucho más alto que el promedio del grupo 1 lo que indica que, el club Atahualpa no realiza inclinación en la articulación de la rodilla y por ende la velocidad del tiro libre se ve disminuida.

\section{Prueba de Mann-Whitney para la Velocidad}

Tabla 7: Rangos de la variable velocidad del Club Jit (Grupo-A) y Club Atahualpa (Grupo-B)

\begin{tabular}{|r|c|c|c|c|}
\hline \multirow{2}{*}{ DATOS } & GRUPOS & $\mathbf{N}$ & $\begin{array}{c}\text { Rango } \\
\text { promedio }\end{array}$ & $\begin{array}{c}\text { Suma de } \\
\text { rangos }\end{array}$ \\
\cline { 2 - 5 } & GRUPO-A & 15 & 20,20 & 303,00 \\
\hline & GRUPO-B & 15 & 10,80 & 162,00 \\
\hline
\end{tabular}


En la tabla 7 podemos observar el número total de la muestra, el rango del club Jit es de 20,20 mientras que el del club Atahualpa es de 10,80 indicándonos claramente la dispersión que existe al comparar a los dos equipos.

Tabla 8: Análisis de datos de la variable velocidad del Club Jit (Grupo-A) y Club Atahualpa (Grupo-B)

\begin{tabular}{|l|c|}
\hline & Datos \\
\hline U de Mann-Whitney & 42,000 \\
W de Wilcoxon & 162,000 \\
Z & $-2,924$ \\
Sig. asintót. (bilateral) &, 003 \\
Sig. exacta [2*(Sig. unilateral)] &, $003(\mathrm{a})$ \\
\hline
\end{tabular}

Se puede observar que existe una diferencia significativa de 0,003 lo cual nos afirma que los resultados obtenidos no fueron por coincidencia. Cabe resaltar que para llegar a este resultado se utilizó la prueba U de Mann-Whitney.

\section{Prueba de Mann-Whitney para Ángulos de Apertura}

Tabla 9: Rangos de la variable angulos apertura del Club Jit (Grupo-A) y Club Atahualpa (Grupo-B)

\begin{tabular}{|c|l|c|c|c|}
\hline \multirow{2}{*}{ DATOS } & GRUPOS & N & $\begin{array}{c}\text { Rango } \\
\text { promedio }\end{array}$ & $\begin{array}{c}\text { Suma de } \\
\text { rangos }\end{array}$ \\
\cline { 2 - 5 } & GRUPO-A & 15 & 20,03 & 300,50 \\
\cline { 2 - 5 } & GRUPO-B & 15 & 10,97 & 164,50 \\
\cline { 2 - 5 } & Total & $\mathbf{3 0}$ & & \\
\hline
\end{tabular}

Encontramos que el número total de la muestra fueron 30 participantes, el rango del club Jit es de 20,03 mientras que el del club Atahualpa es de 10,97.

Tabla 10: Analisis de datos de la variable angulos apertura del Club Jit (Grupo-A) y Club Atahualpa (Grupo-B)

\begin{tabular}{|l|c|}
\hline & Datos \\
\hline U de Mann-Whitney & 44,500 \\
\hline W de Wilcoxon & 164,500 \\
\hline Z & $-2,830$ \\
\hline Sig. asintót. (bilateral) &, 005 \\
\hline Sig. exacta [2*(Sig. unilateral)] &, $004(\mathrm{a})$ \\
\hline
\end{tabular}


Se observa que existe una diferencia significativa de 0,005. Para analizar los datos se utilizó la prueba U de Mann-Whitney

\section{Prueba de Mann-Whitney para los Ángulos de Rodilla de la pierna base}

Tabla 11: Analisis de datos de la variable angulos de rodilla del Club Jit (Grupo-A) y Club Atahualpa (Grupo-B)

\begin{tabular}{|c|l|c|c|c|}
\hline \multirow{2}{*}{ DATOS } & GRUPOS & $\mathbf{N}$ & $\begin{array}{c}\text { Rango } \\
\text { promedio }\end{array}$ & $\begin{array}{c}\text { Suma de } \\
\text { rangos }\end{array}$ \\
\cline { 2 - 5 } & GRUPO-A & 15 & 10,83 & 162,50 \\
\cline { 2 - 5 } & GRUPO-B & 15 & 20,17 & 302,50 \\
\cline { 2 - 5 } & Total & $\mathbf{3 0}$ & & \\
\hline
\end{tabular}

Encontramos que el número total de la muestra fueron 30 participantes, el rango del club Jit es de 10,83 mientras que el del club Atahualpa es de 20,17.

Tabla 12: Analisis de datos de la variable angulos de rodilla del Club Jit (Grupo-A) y Club Atahualpa (Grupo-B)

\begin{tabular}{|l|c|}
\hline & Datos \\
\hline U de Mann-Whitney & 42,500 \\
\hline W de Wilcoxon & 162,500 \\
\hline Z & $-2,904$ \\
\hline Sig. asintót. (bilateral) &, 004 \\
\hline Sig. exacta [2*(Sig. unilateral)] &, $003(\mathrm{a})$ \\
\hline
\end{tabular}

Se observa que existe una diferencia significativa de 0,004. Para analizar los datos se utilizó la prueba U de Mann-Whitney

En la presente investigación se buscó encontrar las diferencias biomecánicas entre un equipo de fútbol profesional y un equipo de fútbol amateur, para ello se analizaron tres variables, la primera fue la velocidad del pie al momento de impactar con el balón en la ejecución del tiro libre, en el cual se puede evidenciar que los pateadores del equipo JIT de segunda categoría profesional son más veloces, por otro lado también se analizó el ángulo de apertura que adoptan los cobradores de tiros libres. Se encontró que, al formar un mayor ángulo de apertura tomando como referencia los puntos del pie de base, el pie de contacto con el balón y la cadera se logra en la mayoría de los casos una mayor velocidad de 
ejecución del tiro libre y por ende una mayor precisión y mayor efectividad en el tiro libre. En la tercera variable, encontramos que el club JIT tiene menor ángulo en la articulación de la rodilla de la pierna base, a diferencia del club Atahualpa que tiene un número más elevado en estos ángulos, lo que significa que al momento de realizar el tiro libre los jugadores del grupo 1 tienen mayor inclinación lo que les permite generar mayor velocidad en la ejecución de la técnica estudiada. Al realizar un promedio de los resultados obtenidos se puede concluir que para la correcta ejecución del libre la persona debería adoptar un ángulo de apertura no menor a 75,66 y una velocidad de $8,96 \mathrm{~m} / \mathrm{s}$ y un ángulo de rodilla de la pierna base de aproximado $106,81^{\circ}$. En el análisis se puede observar que claramente el equipo profesional es mejor cobrador de tiros libres y esto puede deberse a que su entrenamiento es diario y cuenta con implementos mejores a los del equipo de fútbol Amateur. (Quilachamin \& Torres, 2021)

\section{CONCLUSIONES}

El análisis biomecánico demuestra que existe una diferencia importante en la ejecución de los tiros libres, esto debido al distinto nivel que presentan los grupos estudiados, los jugadores del equipo profesional de la segunda categoría muestra que tiene una mayor eficacia en el desarrollo del elemento técnico, nos basamos en la velocidad de ejecución al momento de conectar el pie con el balón lo que permite tener también una mayor potencia en el disparo; en el club amateur, en sus jugadores, no existe tal velocidad y fuerza, sin embargo, no quita méritos a realizar un tiro libre con un grado de precisión pero sin mayor potencia. Entrenar en el aspecto físico también es importante para poder realizar el tiro libre, se cree que solo influye la parte técnica para una buena ejecución, sin embargo, la fuerza, la velocidad y posteriormente la potencia influye en un entrenamiento compuesto y dirigido a la mejora del jugador como tal, al igual que el lugar de preferencia del deportista, no es lo mismo realizar un tiro libre de larga distancia a uno de media o corta distancia.

El software Kinovea es un programa con diversas herramientas en donde se permite dibujar, medir, tomar la captura, analizar ángulos, velocidades, etc. Esto es de gran ayuda para nosotros como entrenadores que buscamos el mejoramiento del jugador. Basándonos en este programa y con la ayuda del software estadístico SPSS podemos observar que un ángulo correcto para la ejecución del tiro libre estaría entre $75,66^{\circ}$ de apertura entre el pie de apoyo y el pie de impacto con el balón con referencia a la cadera, en cuanto a la velocidad un promedio estaría entre los $8,96 \mathrm{~m} / \mathrm{s}$ y el ángulo idóneo de la articulación de 
la rodilla de la pierna base debería estar en un $106,81^{\circ}$. Mediante el análisis individual de las tablas se puede evidenciar que, a mayor ángulo de apertura, y mayor inclinación en la articulación de la rodilla de la pierna base se logra generar mayor velocidad.

El entorno del jugador influye mucho en la ejecución del elemento técnico, partimos con el entrenamiento que recibe cada uno, los jugadores profesionales por las circunstancias necesarias viven de lleno para el fútbol ya que para muchos de ahí reciben su remuneración, por lo que hay un plus extra en la práctica deportiva, en los jugadores amateurs, no existen los entrenamientos dirigidos de lunes a viernes, por lo general se entrenan pocos días debido a que muchos de ellos tienen otras actividades de mayor relevancia en su vida diaria y que a la larga impide desarrollarse como fútbolista; el espacio en donde se entrena varía entre los equipos, el equipo Juventud Independiente de Tabacundo, tiene su propia cancha de entrenamiento a diferencia del club Sociedad Deportiva Atahualpa, el cual cambia con normalidad su lugar de entrenamiento, lo cual influye en la confianza del jugador y de su espacio de entrenamiento, otro tópico a tomarlo en cuenta es a la calidad de los balones que tiene cada equipo, el equipo profesional cuenta con todos los balones de alta gama, lo que ayuda al buen desarrollo del jugador en la parte técnica; en cambio, el equipo amateur dispone de balones en su mayoría no de la mejor calidad, que sin duda alguna perjudica en el rendimiento del mismo.

\section{REFERENCIAS}

Angos Sánchez, C. I. (Diciembre de 2015). Variación del rango articular de tobillo mediante goniometría en pacientes con esguince de la Clínica Panamericana. [Tesis de Licenciatura]. Pontificia Universidad Católica del Ecuador, Quito - Ecuador. Obtenido de Repositorio PUCE.

Charmant, J. (2004). Kinovea. Recuperado el 07 de junio de 2021, de https://www.kinovea.org/

Dell, M. (s.f.). DELL. Recuperado el 07 de junio de 2021, de https://www.dell.com/ec/p/inspiron-15-5567-laptop/pd

Fernández, P. (27 de diciembre de 2017). SPSS: el software ideal para el análisis estadístico. Recuperado el 07 de junio de 2021, de Universidad Privada TELESUP: 
https://escueladeposgrado.edu.pe/blog/spss-el-software-ideal-para-el-analisisestadistico-y-de-mineria-de-datos/

Ferro, A., \& Floría, P. (2007). La aplicación de la biomecánica al entrenamiento deportivo La aplicación de la biomecánica al entrenamiento deportivo mediante los análisis cualitativo y cuantitativo. Una propuesta mediante los análisis cualitativo y cuantitativo. Una propuesta para el 1. Revista Internacional de Ciencias del Deporte, 3(7), 49-80.

Jordán Sánchez, J. W., Espinoza Álvarez, E. 1., Aguilar Salazar, J. A., Hidalgo Alava, D. J., \& Gutiérrez Cruz, M. (2018). Estudio biomecánico del tiro penal: comparación en futbolistas juveniles y de iniciación. Revista Cubana de Investigaciones Biomédicas, 37(04).

Mantilla, I. (2019). Herramientas tecnológicas para el estudio e intervención de la biomecánica en el deporte de alto rendimiento: una mirada desde fisioterapia. Revista Iberoamericana de Ciencias de la Actividad Física y el Deporte, 8(3), 6778.

Mejia, T. (12 de diciembre de 2019). Investigación correlacional: características, tipos y ejemplos. Recuperado el 07 de junio de 2021, de Lifeder: https://www.lifeder.com/investigacion-correlacional/

Núñez Garrido, J., \& Briones Quiroz, F. M. (2015). Fútbol amateur como herramienta de ascenso social. [Título de profesor]. [Universidad del Bío-Bío, Chillán - Chile.

Paez, F. (2010). Biomecánica, herramienta ideal para deportistas. Recuperado el 07 de junio de 2021, de Biomec: http://www.biomec.com.co/Biomecanicadeportiva.html

Perdomo Ogando, J. M., Pegudo Sánchez, A. G., \& Capote Dominguez, T. E. (2018). Premisas para la investigación biomecánica en la cultura física. Revista Cubana Educación Superior, 37(2), 104-114.

Quilachamin, O., \& Torres, M. (07 de junio de 2021). DIFERENCIAS BIOMECÁNICAS DEL TIRO LIBRE EN EL FÚTBOL ENTRE JUGADORES DEL CLUB JIT Y ATAHUALPA. Quito, Pichincha, Ecuador.

Rodríguez, J. (2011). Contrato de gerenciamiento deportivo entre equipos de fútbol y sociedades anónimas según la legislación ecuatoriana. Quito: Pontificia Universidad Católica del Ecuador. 
Roffé, M. (septiembre de 2015). El Psicólogo del Deporte en el Fútbol Amateur: La Prevención. Lecturas: Educación Física y Deportes. Buenos Aires: Revista Digital, $1-7$.

Samsung. (2016). Galaxy S7. Recuperado el 07 de junio de 2021, de https://www.samsung.com/es/smartphones/galaxy-s7-g930f/SM-G930FZKAPHE/

Sánchez Pay, A. (2018). El uso del kinovea para el análisis biomecánico desde una perspectiva cuantitativa. TRANCES: Revista de Transmisión del Conocimiento Educativo y de la Salud, 6(4), 725-738.

Vásquez Castillo, N. A., \& Montesinos Guarnizo, L. B. (2016). Las técnicas del fútbol el tiro a portería y el dribling en los niños de 10 a 12 del cantón Sozoranga de la provincia de Loja. [Tesis de Licenciatura]. Universidad Nacional de Loja, Loja Ecuador. 


\section{ANEXOS}
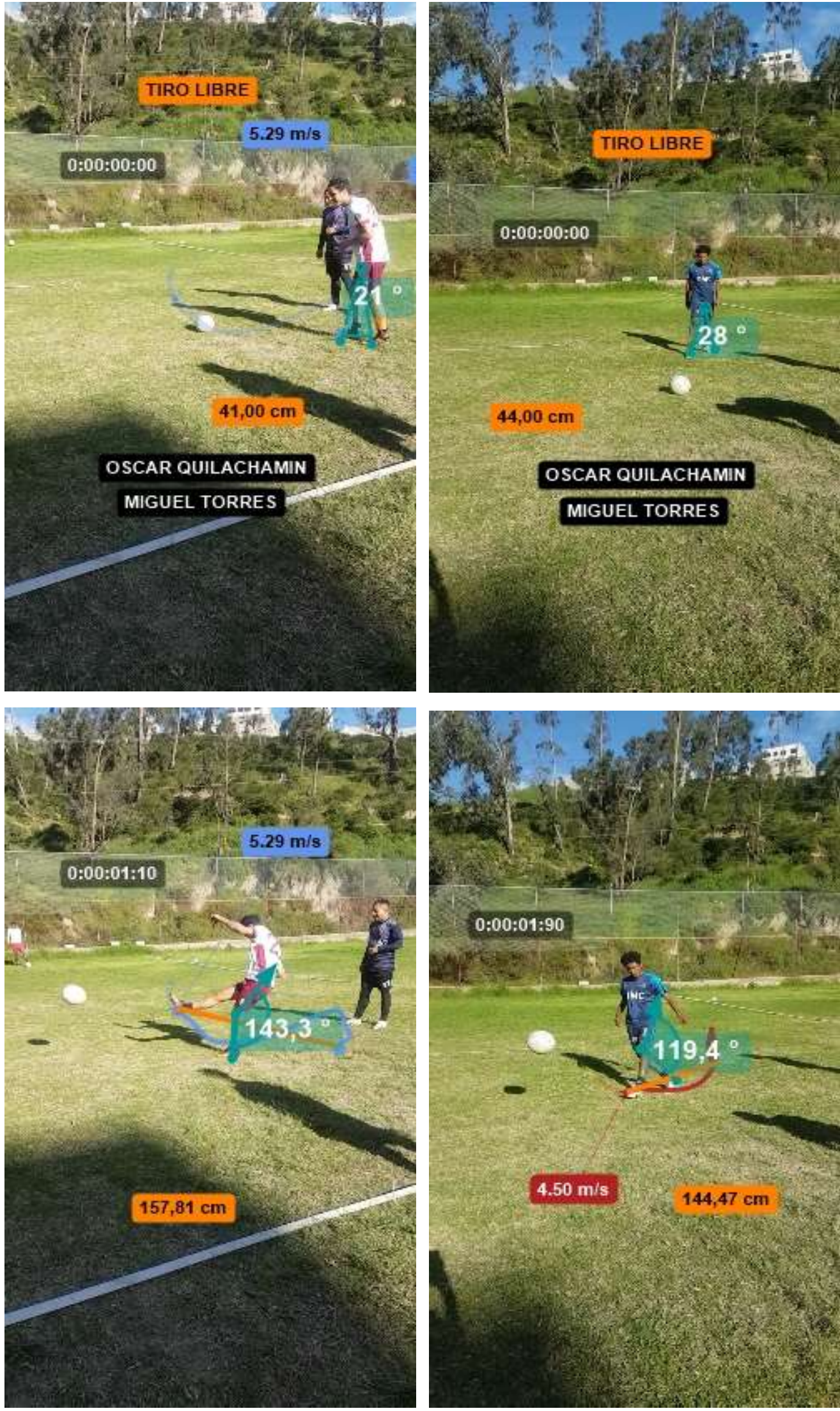

Ciencia Latina Revista Científica Multidisciplinar, Ciudad de México, México. ISSN 2707-2207 / ISSN 2707-2215 (en línea), julio-agosto, 2021, Volumen 5, Número 4. https://doi.org/10.37811/cl rcm.v5i4.635 p.4536 
Quilachamin Espinoza, Torres Morillo y Coral Apolo
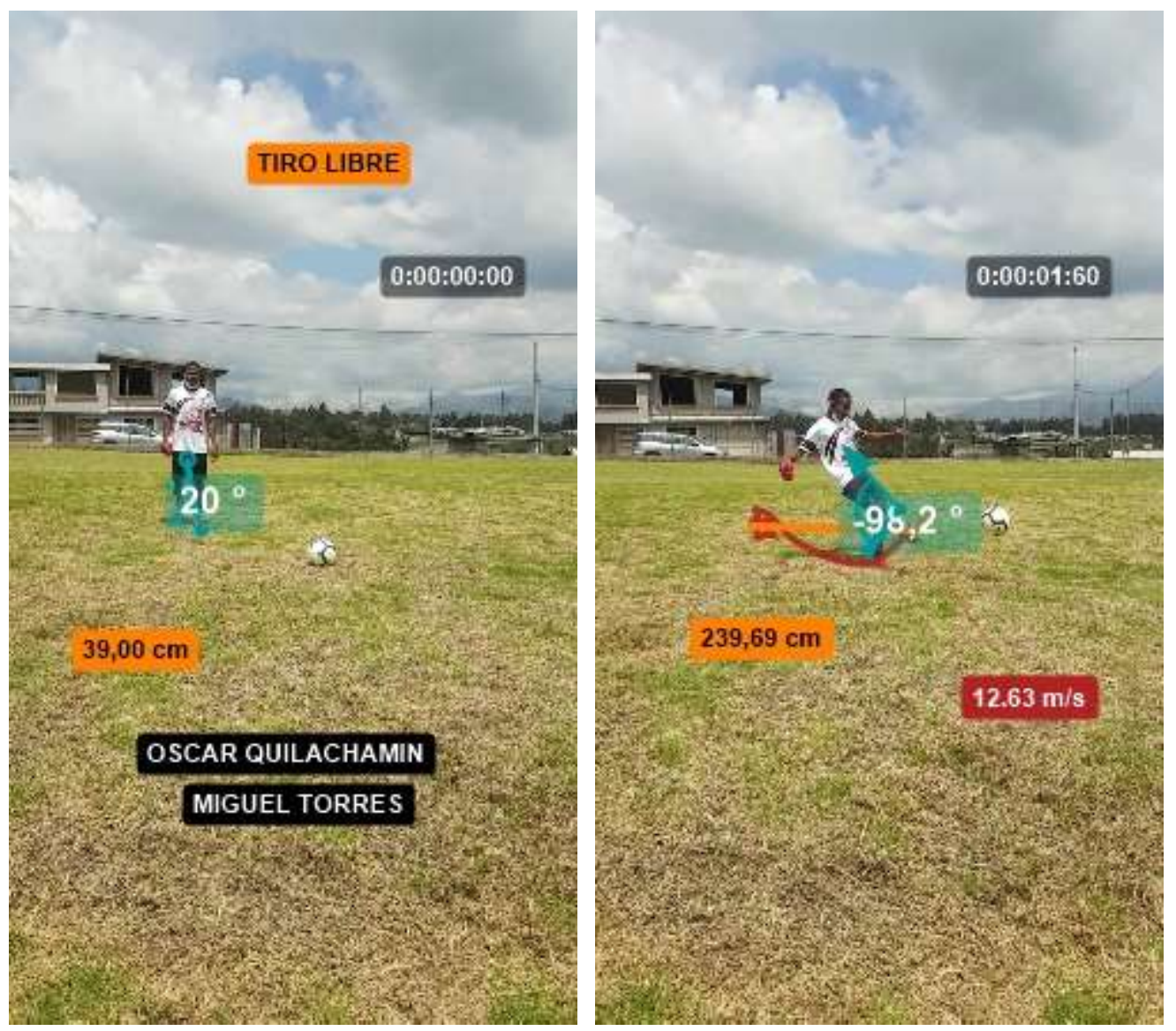

Ciencia Latina Revista Científica Multidisciplinar, Ciudad de México, México. ISSN 2707-2207 / ISSN 2707-2215 (en línea), julio-agosto, 2021, Volumen 5, Número 4. https://doi.org/10.37811/cl rcm.v5i4.635 p.4537 

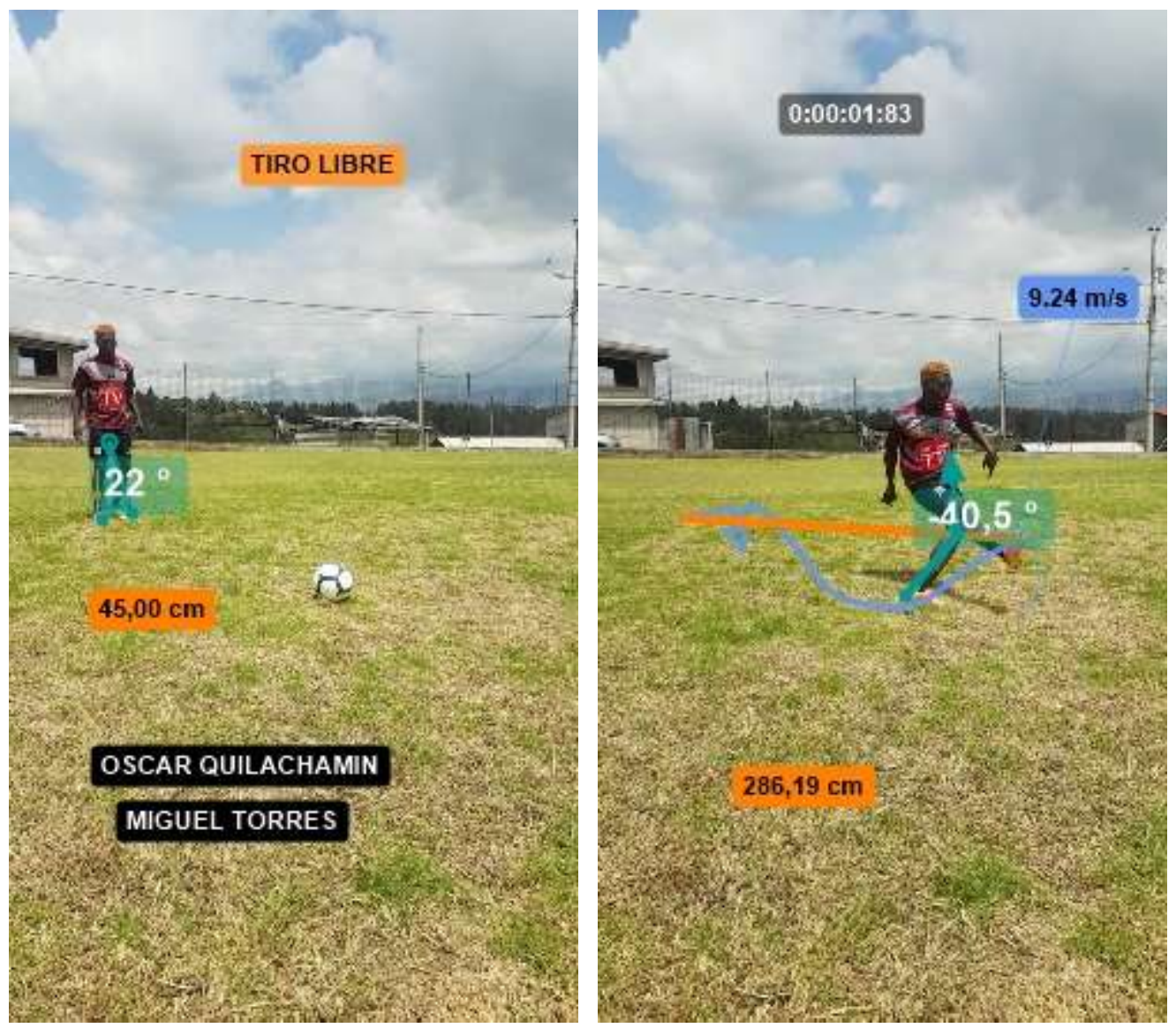

Ciencia Latina Revista Científica Multidisciplinar, Ciudad de México, México. ISSN 2707-2207 / ISSN 2707-2215 (en línea), julio-agosto, 2021, Volumen 5, Número 4. https://doi.org/10.37811/cl rcm.v5i4.635 p.4538 